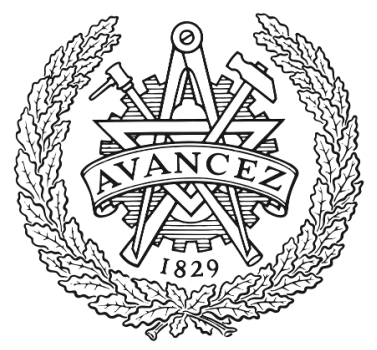

CHALMERS

UNIVERSITY OF TECHNOLOGY

\title{
Branching Processes for QuickCheck Generators
}

Downloaded from: https://research.chalmers.se, 2023-04-26 10:11 UTC

Citation for the original published paper (version of record):

Mista, C., Russo, A., Hughes, J. (2018). Branching Processes for QuickCheck Generators. Haskell 2018 - Proceedings of the 11th ACM SIGPLAN International Symposium on Haskell, co-located with ICFP 2018, 53(7): 1-13. http://dx.doi.org/10.1145/3242744.3242747

N.B. When citing this work, cite the original published paper. 


\section{Branching Processes for QuickCheck Generators}

\author{
Agustín Mista \\ Universidad Nacional de Rosario \\ Rosario, Argentina \\ amista@dcc.fceia.unr.edu.ar
}

\author{
Alejandro Russo \\ Chalmers University of Technology \\ Gothenburg, Sweden \\ russo@chalmers.se
}

\author{
John Hughes \\ Chalmers University of Technology \\ Gothenburg, Sweden \\ rjmh@chalmers.se
}

\begin{abstract}
In QuickCheck (or, more generally, random testing), it is challenging to control random data generators' distributionsspecially when it comes to user-defined algebraic data types (ADT). In this paper, we adapt results from an area of mathematics known as branching processes, and show how they help to analytically predict (at compile-time) the expected number of generated constructors, even in the presence of mutually recursive or composite ADTs. Using our probabilistic formulas, we design heuristics capable of automatically adjusting probabilities in order to synthesize generators which distributions are aligned with users' demands. We provide a Haskell implementation of our mechanism in a tool called DRAGEN and perform case studies with real-world applications. When generating random values, our synthesized QuickCheck generators show improvements in code coverage when compared with those automatically derived by state-of-the-art tools.
\end{abstract}

CCS Concepts - Software and its engineering $\rightarrow$ Software testing and debugging;

Keywords Branching process, QuickCheck, Testing, Haskell

ACM Reference Format:

Agustín Mista, Alejandro Russo, and John Hughes. 2018. Branching Processes for QuickCheck Generators. In Proceedings of the 11th ACM SIGPLAN International Haskell Symposium (Haskell '18), September 27-28, 2018, St. Louis, MO, USA. ACM, New York, NY, USA, 13 pages. https://doi.org/10.1145/3242744.3242747

\section{Introduction}

Random property-based testing is an increasingly popular approach to finding bugs $[3,16,17]$. In the Haskell community, QuickCheck [9] is the dominant tool of this sort. QuickCheck requires developers to specify testing properties describing the expected software behavior. Then, it generates a large number

Permission to make digital or hard copies of all or part of this work for personal or classroom use is granted without fee provided that copies are not made or distributed for profit or commercial advantage and that copies bear this notice and the full citation on the first page. Copyrights for components of this work owned by others than the author(s) must be honored. Abstracting with credit is permitted. To copy otherwise, or republish, to post on servers or to redistribute to lists, requires prior specific permission and/or a fee. Request permissions from permissions@acm.org.

Haskell '18, September 27-28, 2018, St. Louis, MO, USA

(c) 2018 Copyright held by the owner/author(s). Publication rights licensed to ACM.

ACM ISBN 978-1-4503-5835-4/18/09 .. \$15.00

https://doi.org/10.1145/3242744.3242747 of random test cases and reports those violating the testing properties. QuickCheck generates random data by employing random test data generators or QuickCheck generators for short. The generation of test cases is guided by the types involved in the testing properties. It defines default generators for many built-in types like booleans, integers, and lists. However, when it comes to user-defined ADTs, developers are usually required to specify the generation process. The difficulty is, however, that it might become intricate to define generators so that they result in a suitable distribution or enforce data invariants.

The state-of-the-art tools to derive generators for userdefined ADTs can be classified based on the automation level as well as the sort of invariants enforced at the data generation phase. QuickCheck and SmallCheck [27] (a tool for writing generators that synthesize small test cases) use type-driven generators written by developers. As a result, generated random values are well-typed and preserve the structure described by the ADT. Rather than manually writing generators, libraries derive [24] and MegaDeTH [13, 14] automatically synthesize generators for a given user-defined ADT. The library derive provides no guarantees that the generation process terminates, while MegaDeTH pays almost no attention to the distribution of values. In contrast, Feat [11] provides a mechanism to uniformly sample values from a given ADT. It enumerates all the possible values of a given ADT so that sampling uniformly from ADTs becomes sampling uniformly from the set of natural numbers. Feat's authors subsequently extend their approach to uniformly generate values constrained by userdefined predicates [8]. Lastly, Luck is a domain specific language for manually writing QuickCheck properties in tandem with generators so that it becomes possible to finely control the distribution of generated values [18].

In this work, we consider the scenario where developers are not fully aware of the properties and invariants that input data must fulfill. This constitutes a valid assumption for penetration testing [2], where testers often apply fuzzers in an attempt to make programs crash-an anomaly which might lead to a vulnerability. We believe that, in contrast, if users can recognize specific properties of their systems then it is preferable to spend time writing specialized generators for that purpose (e.g., by using Luck) instead of considering automatically derived ones.

Our realization is that branching processes [29], a relatively simple stochastic model conceived to study the evolution of populations, can be applied to predict the generation distribution of ADTs' constructors in a simple and automatable 
manner. To the best of our knowledge, this stochastic model has not yet been applied to this field, and we believe it may be a promising foundation to develop future extensions. The contributions of this paper can be outlined as follows:

- We provide a mathematical foundation which helps to analytically characterize the distribution of constructors in derived QuickCheck generators for ADTs.

- We show how to use type reification to simplify our prediction process and extend our model to mutually recursive and composite types.

- We design (compile-time) heuristics that automatically search for probability parameters so that distributions of constructors can be adjusted to what developers might want.

- We provide an implementation of our ideas in the form of a Haskell library ${ }^{1}$ called DRAGEN (the Danish word for dragon, here standing for Derivation of RAndom GENerators).

- We evaluate our tool by generating inputs for real-world programs, where it manages to obtain significantly more code coverage than those random inputs generated by MegaDeTH's generators.

Overall, our work addresses a timely problem with a neat mathematical insight that is backed by a complete implementation and experience on third-party examples.

\section{Background}

In this section, we briefly illustrate how QuickCheck random generators work. We consider the following implementation of binary trees:

\section{data Tree $=$ Leaf $_{A} \mid$ Leaf $_{B} \mid$ Leaf $_{C} \mid$ Node Tree Tree}

In order to help developers write generators, QuickCheck defines the Arbitrary type-class with the overloaded symbol arbitrary :: Gen a, which denotes a monadic generator for values of type $a$. Then, to generate random trees, we need to provide an instance of the Arbitrary type-class for the type Tree. Figure 1 shows a possible implementation. At the top level, this generator simply uses QuickCheck's primitive oneof :: [ [ Gen a $] \rightarrow$ Gen a to pick a generator from a list of generators with uniform probability. This list consists of a random generator for each possible choice of data constructor of Tree. We use applicative style [21] to describe each one of them idiomatically. So, pure Leaf $_{A}$ is a generator that always generates Lea $f_{A} \mathrm{~s}$, while Node $\langle \$\rangle$ arbitrary $\langle *\rangle$ arbitrary is a generator that always generates Node constructors, "filling" its arguments by calling arbitrary recursively on each of them.

\footnotetext{
${ }^{1}$ Available at https://bitbucket.org/agustinmista/dragen

instance Arbitrary Tree where arbitrary $=$ oneof $\left[\right.$ pure Lea $f_{A}$, pure Lea $f_{B}$, pure Lea $f_{C}$ , Node $\langle \$\rangle$ arbitrary $\langle *\rangle$ arbitrary]
}

Figure 1. Random generator for Tree.
Although it might seem easy, writing random generators becomes cumbersome very quickly. Particularly, if we want to write a random generator for a user-defined ADT $T$, it is also necessary to provide random generators for every userdefined ADT inside of $T$ as well! What remains of this section is focused on explaining the state-of-the-art techniques used to automatically derive generators for user-defined ADTs via type-driven approaches.

\subsection{Library derive}

The simplest way to automatically derive a generator for a given ADT is the one implemented by the Haskell library derive [24]. This library uses Template Haskell [28] to automatically synthesize a generator for the data type Tree semantically equivalent to the one presented in Figure 1.

While the library derive is a big improvement for the testing process, its implementation has a serious shortcoming when dealing with recursively defined data types: in many cases, there is a non-zero probability of generating a recursive type constructor every time a recursive type constructor gets generated, which can lead to infinite generation loops. A detailed example of this phenomenon is presented in the supplementary material [23]. In this work, we only focus on derivation tools which accomplish terminating behavior, since we consider this an essential component of well-behaved generators.

\subsection{MegaDeTH}

The second approach we will discuss is the one taken by MegaDeTH, a meta-programming tool used intensively by QuickFuzz [13, 14]. Firstly, MegaDeTH derives random generators for ADTs as well as all of its nested types-a useful feature not supported by derive. Secondly, MegaDeTH avoids potentially infinite generation loops by setting an upper bound to the random generation recursive depth.

Figure 2 shows a simplified (but semantically equivalent) version of the random generator for Tree derived by MegaDeTH. This generator uses QuickCheck's function sized :: (Int $\rightarrow$ Gen $a) \rightarrow$ Gen $a$ to build a random generator based on a function (of type Int $\rightarrow$ Gen a) that limits the possible recursive calls performed when creating random values. The integer passed to sized's argument is called the generation size. When the generation size is zero (see definition gen 0 ), the generator only chooses between the Tree's terminal constructors-thus

$$
\begin{aligned}
& \text { instance Arbitrary Tree where } \\
& \text { arbitrary }=\text { sized gen where } \\
& \text { gen } 0=\text { oneof } \\
& {\left[\text { pure Lea } f_{A} \text {, pure Lea } f_{B} \text {, pure Lea } f_{C}\right]} \\
& \text { gen } n=\text { oneof } \\
& {\left[\text { pure Leaf } f_{A} \text {, pure Leaf } f_{B}, \text { pure Leaf } f_{C}\right.} \\
& \text {,Node }\langle \$\rangle \text { gen }(\text { div } n 2)\langle *\rangle \text { gen }(\operatorname{div} n 2)]
\end{aligned}
$$

Figure 2. MegaDeTH generator for Tree. 
ending the generation process. If the generation size is strictly positive, it is free to randomly generate any Tree constructor (see definition gen $n$ ). When it chooses to generate a recursive constructor, it reduces the generation size for its subsequent recursive calls by a factor that depends on the number of recursive arguments this constructor has ( $\operatorname{div} n 2$ ). In this way, MegaDeTH ensures that all generated values are finite.

Although MegaDeTH generators always terminate, they have a major practical drawback: in our example, the use of oneof to uniformly decide the next constructor to be generated produces a generator that generates leaves approximately three quarters of the time (note this also applies to the generator obtained with derive from Figure 1). This entails a distribution of constructors heavily concentrated on leaves, with a very small number of complex values with nested nodes, regardless how large the chosen generation size is-see Figure 3 (left).

\subsection{Feat}

The last approach we discuss is Feat [11]. This tool determines the distribution of generated values in a completely different way: it uses uniform generation based on an exhaustive enumeration of all the possible values of the ADTs being considered. Feat automatically establishes a bijection between all the possible values of a given type $T$, and a finite prefix of the natural numbers. Then, it guarantees a uniform generation over the complete space of values of a given data type $T$ up to a certain size. ${ }^{2}$ However, the distribution of size, given by the number of constructors in the generated values, is highly dependent on the structure of the data type being considered.

Figure 3 (right) shows the overall distribution shape of a QuickCheck generator derived using Feat for Tree using a generation size of 400 , i.e., generating values of up to 400 constructors. ${ }^{3}$ Notice that all the generated values are close to the maximum size! This phenomenon follows from the exponential growth in the number of possible Trees of $n$ constructors as we increase $n$. In other words, the space of Trees up to 400 constructors is composed to a large extent of values with around 400 constructors, and (proportionally) very few with a smaller number of constructors. Hence, a generation process based on uniform generation of a natural number (which thus ignores the structure of the type being generated) is biased very strongly towards values made up of a large number of constructors. In our tests, no Tree with less than 390 constructors was ever generated. In practice, this problem can be partially solved by using a variety of generation sizes in order to get more diversity in the generated values. However, to decide which generation sizes are the best choices is not a trivial task either. As consequence, in this work we consider only the case of fixed-size random generation.

\footnotetext{
${ }^{2}$ We avoid including any source code generated by Feat, since it works by synthetizing Enumerable type-class instances instead of Arbitrary ones. Such instances give no insight into how the derived random generators work.

${ }^{3}$ We choose to use this generation size here since it helps us to compare MegaDeTH and Feat with the results of our tool in Section 8.
}
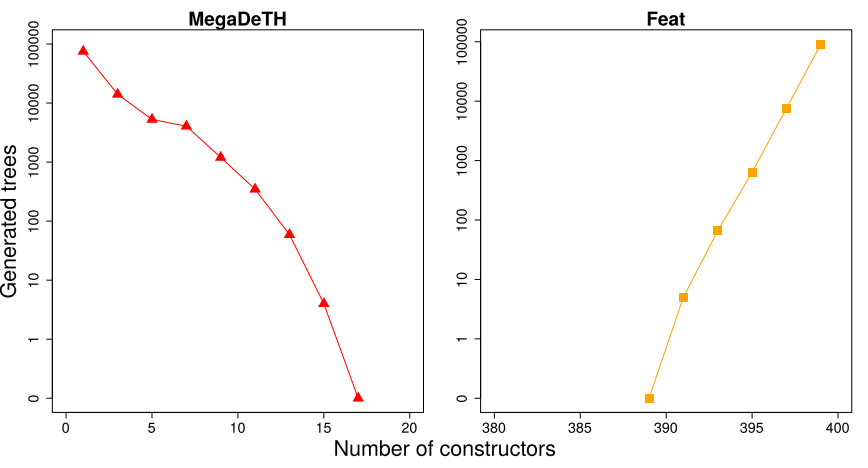

Figure 3. Size distribution of 100000 randomly generated Tree values using MegaDeTH ( $\mathbf{\Delta})$ with generation size 10, and Feat $(\square)$ with generation size 400 .

As we have shown, by using both MegaDeTH and Feat, the user is tied to the fixed generation distribution that each tool produces, which tends to be highly dependent on the particular data type under consideration on each case. Instead, this work aims to provide a theoretical framework able to predict and later tune the distributions of automatically derived generators, giving the user a more flexible testing environment, while keeping it as automated as possible.

\section{Simple-Type Branching Processes}

Galton-Watson Branching processes (or branching processes for short) are a particular case of Markov processes that model the growth and extinction of populations. Originally conceived to study the extinction of family names in the Victorian era, this formalism has been successfully applied to a wide range of research areas in biology and physics-see the textbook by Haccou et al. [15] for an excellent introduction. In this section, we show how to use this theory to model QuickCheck's distribution of constructors.

We start by analyzing the generation process for the Node constructors in the data type Tree as described by the generators in Figure 1 and 2. From the code, we can observe that the stochastic process they encode satisfies the following assumptions (which coincide with the assumptions of Galton-Watson branching processes): i) With a certain probability, it starts with some initial Node constructor. ii) At any step, the probability of generating a Node is not affected by the Nodes generated before or after. iii) The probability of generating a Node is independent of where in the tree that constructor is about to be placed.

The original Galton-Watson process is a simple stochastic process that counts the population sizes at different points in time called generations. For our purposes, populations consist of Node constructors, and generations are obtained by selecting tree levels.

Figure 4 illustrates a possible generated value. It starts by generating a Node constructor at generation (i.e., depth) zero $\left(G_{0}\right)$, then another two Node constructors as left and right 
subtrees in generation one $\left(G_{1}\right)$, etc. (Dotted edges denote further constructors which are not drawn, as they are not essential for the point being made.) This process repeats until the population of Node constructors becomes extinct or stable, or alternatively grows forever.

The mathematics behind the Galton-Watson process allows us to predict the expected number of offspring at the $n$ th-generation, i.e., the number of Node constructors at depth $n$ in the generated tree. Formally, we start by introducing the random variable $R$ to denote the number of Node con-

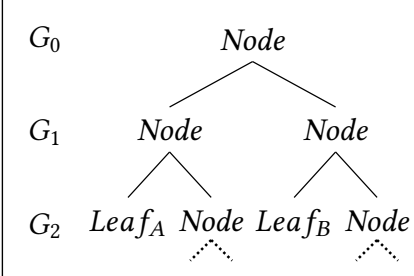

Figure 4. Generation of Node constructors. structors in the next generation generated by a Node constructor in this generation-the $R$ comes from "reproduction" and the reader can think it as a Node constructor reproducing Node constructors. To be a bit more general, let us consider the Tree random generator automatically generated using $d e-$ rive (Figure 1), but where the probability of choosing between any constructor is no longer uniform. Instead, we have a $p_{C}$ probability of choosing the constructor $C$. These probabilities are external parameters of the prediction mechanism, and Section 7 explains how they can later be instantiated with actual values found by optimization, enabling the user to tune the generated distribution.

We note $p_{\text {Leaf }}$ as the probability of generating a leaf of any kind, i.e., $p_{\text {Leaf }}=p_{\text {Leaf } A}+p_{\text {Leaf } B}+p_{\text {LeafC } C}$. In this setting, and assuming a parent constructor Node, the probabilities of generating $R$ numbers of Node offspring in the next generation (i.e., in the recursive calls of arbitrary) are as follows:

$$
\begin{aligned}
& P(R=0)=p_{\text {Leaf }} \cdot p_{\text {Leaf }} \\
& P(R=1)=p_{\text {Node }} \cdot p_{\text {Leaf }}+p_{\text {Leaf }} \cdot p_{\text {Node }}=2 \cdot p_{\text {Node }} \cdot p_{\text {Leaf }} \\
& P(R=2)=p_{\text {Node }} \cdot p_{\text {Node }}
\end{aligned}
$$

One manner to understand the equations above is by considering what QuickCheck does when generating the subtrees of a given node. For instance, the cases when generating exactly one Node as descendant $(P(R=1))$ occurs in two situations: when the left subtree is a Node and the right one is a Leaf; and viceversa. The probability for those events to occur is $p_{\text {Node }} * p_{\text {Leaf }}$ and $p_{\text {Leaf }} * p_{\text {Node }}$, respectively. Then, the probablity of having exactly one Node as a descendant is given by the sum of the probability of both events-the other cases follow a similar reasoning.

Now that we have determined the distribution of $R$, we proceed to introduce the random variables $G_{n}$ to denote the population of Node constructors in the $n$th generation. We write $\xi_{i}^{n}$ for the random variable which captures the number of (offspring) Node constructors at the $n$th generation produced by the $i$ th Node constructor at the (n-1)th generation. It is easy to see that it must be the case that $G_{n}=\xi_{1}^{n}+\xi_{2}^{n}+\cdots+\xi_{G_{n-1}}^{n}$. To deduce $E\left[G_{n}\right]$, i.e. the expected number of Nodes in the $n$th generation, we apply the (standard) Law of Total Expectation $E[X]=E[E[X \mid Y]]^{4}$ with $X=G_{n}$ and $Y=G_{n-1}$ to obtain:

$$
E\left[G_{n}\right]=E\left[E\left[G_{n} \mid G_{n-1}\right]\right] .
$$

By expanding $G_{n}$, we deduce that:

$$
\begin{aligned}
E\left[G_{n} \mid G_{n-1}\right] & =E\left[\xi_{1}^{n}+\xi_{2}^{n}+\cdots+\xi_{G_{n-1}}^{n} \mid G_{n-1}\right] \\
& =E\left[\xi_{1}^{n} \mid G_{n-1}\right]+E\left[\xi_{2}^{n} \mid G_{n-1}\right]+\cdots+E\left[\xi_{G_{n-1}}^{n} \mid G_{n-1}\right]
\end{aligned}
$$

Since $\xi_{1}^{n}, \xi_{2}^{n}, \ldots$, and $\xi_{G_{n-1}}^{n}$ are all governed by the distribution captured by the random variable $R$ (recall the assumptions at the beginning of the section), we have that:

$$
E\left[G_{n} \mid G_{n-1}\right]=E\left[R \mid G_{n-1}\right]+E\left[R \mid G_{n-1}\right]+\cdots+E\left[R \mid G_{n-1}\right]
$$

Since $R$ is independent of the generation where Node constructors decide to generate other Node constructors, we have that

$$
E\left[G_{n} \mid G_{n-1}\right]=\underbrace{E[R]+E[R]+\cdots+E[R]}_{G_{n-1} \text { times }}=E[R] \cdot G_{n-1}
$$

From now on, we introduce $m$ to denote the mean of $R$, i.e., the mean of reproduction. Then, by rewriting $m=E[R]$, we obtain:

$$
E\left[G_{n}\right] \stackrel{(1)}{=} E\left[E\left[G_{n} \mid G_{n-1}\right]\right] \stackrel{(2)}{=} E\left[m \cdot G_{n-1}\right] \stackrel{\mathrm{m} \text { is constant }}{=} E\left[G_{n-1}\right] \cdot m
$$

By unfolding this recursive equation many times, we obtain:

$$
E\left[G_{n}\right]=E\left[G_{0}\right] \cdot m^{n}
$$

As the equation indicates, the expected number of Node constructors at the $n$th generation is affected by the mean of reproduction. Although we obtained this intuitive result using a formalism that may look overly complex, it is useful to understand the methodology used here. In the next section, we will derive the main result of this work following the same reasoning line under a more general scenario.

We can now also predict the total expected number of individuals up to the $n$th generation. For that purpose, we introduce the random variable $P_{n}$ to denote the population of Node constructors up to the $n$th generation. It is then easy to see that $P_{n}=\sum_{i=0}^{n} G_{i}$ and consequently:

$$
E\left[P_{n}\right]=\sum_{i=0}^{n} E\left[G_{i}\right] \stackrel{(3)}{=} \sum_{i=0}^{n} E\left[G_{0}\right] \cdot m^{i}=E\left[G_{0}\right] \cdot\left(\frac{1-m^{n+1}}{1-m}\right)
$$

where the last equality holds by the geometric series definition. This is the general formula provided by the Galton-Watson process. In this case, the mean of reproduction for Node is given by:

$$
m=E[R]=\sum_{k=0}^{2} k \cdot P(R=k)=2 \cdot p_{\text {Node }}
$$

\footnotetext{
${ }^{4} E[X \mid Y]$ is a function on the random variable $Y$, i.e., $E[X \mid Y] y=E[X \mid Y=y]$ and therefore it is a random variable itself. In this light, the law says that if we observe the expectations of $X$ given the different $y_{s}$, and then we do the expectation of all those values, then we have the expectation of $X$.
} 
By (4) and (5), the expected number of Node constructors up to generation $n$ is given by the following formula:

$$
E\left[P_{n}\right]=E\left[G_{0}\right] \cdot\left(\frac{1-m^{n+1}}{1-m}\right)=p_{\text {Node }} \cdot\left(\frac{1-\left(2 \cdot p_{\text {Node }}\right)^{n+1}}{1-2 \cdot p_{\text {Node }}}\right)
$$

If we apply the previous formula to predict the distribution of constructors induced by MegaDeTH in Figure 2, where $p_{\text {Leaf } A}=p_{\text {Leaf } B}=p_{\text {Leaf } C}=p_{\text {Node }}=0.25$, we obtain an expected number of Node constructors up to level 10 of 0.4997 , which denotes a distribution highly biased towards small values, since we can only produce further subterms by producing Nodes. However, if we set $p_{\text {Leaf } A}=p_{\text {Leaf } B}=p_{\text {Leaf } C}=0.1$ and $p_{N o d e}=0.7$, we can predict that, as expected, our general random generator will generate much bigger trees, containing an average number of 69.1173 Nodes up to level 10! Unfortunately, we cannot apply this reasoning to predict the distribution of constructors for derived generators for ADTs with more than one non-terminal constructor. For instance, let us consider the following data type definition:

\section{data Tree $^{\prime}=$ Leaf $\mid$ Node $_{A}$ Tree $^{\prime}$ Tree $^{\prime} \mid$ Node $_{B}$ Tree $^{\prime}$}

In this case, we need to separately consider that a $\operatorname{Node}_{A}$ can generate not only $\operatorname{Node}_{A}$ but also Node $_{B}$ offspring (similarly with $\operatorname{Node}_{B}$ ). A stronger mathematical formalism is needed. The next section explains how to predict the generation of this kind of data types by using an extension of Galton-Waston processes known as multi-type branching processes.

\section{Multi-Type Branching Processes}

In this section, we present the basis for our main contribution: the application of multi-type branching processes to predict the distribution of constructors. We will illustrate the technique by considering the Tree' ADT that we concluded with in the previous section.

Before we dive into technicalities, Figure 5 shows the automatically derived generator for Tree ${ }^{\prime}$ that our tool produces. Our generators depend on the (possibly) different probabilities that constructors have to be generated-variables $p_{\text {Leaf }}$, $p_{\text {Node } A}$, and $p_{\text {NodeB }}$. These probabilities are used by the function chooseWith :: [(Double, Gen $a)] \rightarrow$ Gen $a$, which picks a random generator of type $a$ with an explicitly given probability from a list. This function can be easily expressed by using QuickCheck's primitive operations and therefore we omit its

instance Arbitrary Tree' where

arbitrary $=$ sized gen where

gen $0=$ pure Leaf

gen $n=$ chooseWith

$\left[\left(p_{\text {Leaf }}\right.\right.$, pure Leaf $)$

,$\left(p_{N_{\text {ode }}, \text { Node }_{A}\langle \$\rangle \text { gen }}(n-1)\langle *\rangle\right.$ gen $\left.(n-1)\right)$

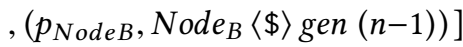

Figure 5. DRAGEN generator for Tree' implementation. Additionally note that, like MegaDeTH, our generators use sized to limit the number of recursive calls to ensure termination. We note that the theory behind branching processes is able to predict the termination behavior of our generators and we could have used this ability to ensure their termination without the need of a depth limiting mechanism like sized. However, using sized provides more control over the obtained generator distributions.

To predict the distribution of constructors provided by DRAGEN generators, we introduce a generalization of the previous Galton-Watson branching process called multi-type GaltonWatson branching process. This generalization allows us to consider several kinds of individuals, i.e., constructors in our setting, to procreate (generate) different kinds of offspring (constructors). Additionally, this approach allows us to consider not just one constructor, as we did in the previous section, but rather to consider all of them at the same time.

Before we present the mathematical foundations, which follow a similar line of reasoning as that in Section 3, Figure 6 illustrates a possible generated value of type Tree'.

In the generation process, it is assumed that the kind (i.e., the constructor) of the parent might affect the probabilities of reproducing (generating) offspring of a certain kind. Observe that this is the case for a wide range of derived ADT generators, e.g., choosing a terminal constructor (e.g., Leaf) affects

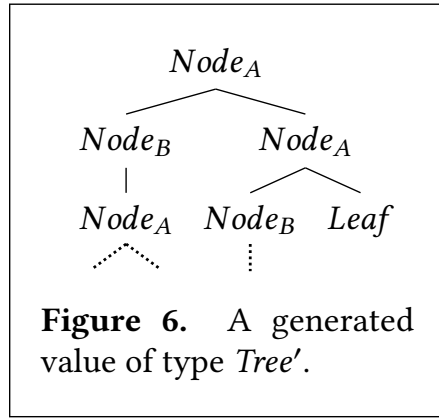
the probabilities of generat-

ing non-terminal ones (by setting them to zero). The population at the $n$th generation is then characterized as a vector of random variables $G_{n}=\left(G_{n}^{1}, G_{n}^{2}, \cdots, G_{n}^{d}\right)$, where $d$ is the number of different kinds of constructors. Each random variable $G_{n}^{i}$ captures the number of occurrences of the $i$ th-constructor of the ADT at the $n$th generation. Essentially, $G_{n}$ "groups" the population at level $n$ by the constructors of the ADT. By estimating the expected shape of the vector $G_{n}$, it is possible to obtain the expected number of constructors at the $n$th generation. Specifically, we have that $E\left[G_{n}\right]=\left(E\left[G_{n}^{1}\right], E\left[G_{n}^{2}\right], \cdots, E\left[G_{n}^{d}\right]\right)$. To deduce $E\left[G_{n}\right]$, we focus on deducing each component of the vector.

As explained above, the reproduction behavior is determined by the kind of the individual. In this light, we introduce random variable $R_{i j}$ to denote a parent $i$ th constructor reproducing a $j$ th constructor. As we did before, we apply the equation $E[X]=E[E[X \mid Y]]$ with $X=G_{n}^{j}$ and $Y=G_{n-1}$ to obtain $E\left[G_{n}^{j}\right]=E\left[E\left[G_{n}^{j} \mid G_{n-1}\right]\right]$. To calculate the expected number of $j$ th constructors at the level $n$ produced by the constructors present at level $(n-1)$, i.e., $E\left[G_{n}^{j} \mid G_{(n-1)}\right]$, it is enough to count the expected number of children of kind $j$ produced by the 
different parents of kind $i$, i.e., $E\left[R_{i j}\right]$, times the amount of parents of kind $i$ found in the level $(n-1)$, i.e., $G_{(n-1)}^{i}$. This result is expressed by the following equation marked as $(\star)$, and is formally verified in the supplementary material.

$$
E\left[G_{n}^{j} \mid G_{n-1}\right] \stackrel{(\star)}{=} \sum_{i=1}^{d} G_{(n-1)}^{i} \cdot E\left[R_{i j}\right]=\sum_{i=1}^{d} G_{(n-1)}^{i} \cdot m_{i j}
$$

Similarly as before, we rewrite $E\left[R_{i j}\right]$ as $m_{i j}$, which now represents a single expectation of reproduction indexed by the kind of both the parent and child constructor.

Mean matrix of constructors In the previous section, $m$ was the expectation of reproduction of a single constructor. Now we have $m_{i j}$ as the expectation of reproduction indexed by the parent and child constructor. In this light, we define $M_{C}$, the mean matrix of constructors (or mean matrix for simplicity) such that each $m_{i j}$ stores the expected number of $j$ th constructors generated by the $i$ th constructor. $M_{C}$ is a parameter of the Galton-Watson multi-type process and can be built at compile-time using statically known type information. We are now able to deduce $E\left[G_{n}^{j}\right]$.

$$
\begin{aligned}
E\left[G_{n}^{j}\right] & =E\left[E\left[G_{n}^{j} \mid G_{n-1}\right]\right] \stackrel{(6)}{=} E\left[\sum_{i=1}^{d} G_{(n-1)}^{i} \cdot m_{i j}\right] \\
& =\sum_{i=1}^{d} E\left[G_{(n-1)}^{i} \cdot m_{i j}\right]=\sum_{i=1}^{d} E\left[G_{(n-1)}^{i}\right] \cdot m_{i j}
\end{aligned}
$$

Using this last equation, we can rewrite $E\left[G_{n}\right]$ as follows.

$$
E\left[G_{n}\right]=\left(\sum_{i=1}^{d} E\left[G_{(n-1)}^{1}\right] \cdot m_{i 1}, \cdots, \sum_{i=1}^{d} E\left[G_{(n-1)}^{d}\right] \cdot m_{i d}\right)
$$

By linear algebra, we can rewrite the vector above as the matrix multiplication $E\left[G_{n}\right]^{T}=E\left[G_{n-1}\right]^{T} \cdot M_{C}$. By repeatedly unfolding this definition, we obtain that:

$$
E\left[G_{n}\right]^{T}=E\left[G_{0}\right]^{T} \cdot\left(M_{C}\right)^{n}
$$

This equation is a generalization of (3) when considering many constructors. As we did before, we introduce a random variable $P_{n}=\sum_{i=0}^{n} G_{i}$ to denote the population up to the $n$th generation It is now possible to obtain the expected population of all the constructors but in a clustered manner:

$$
E\left[P_{n}\right]^{T}=E\left[\sum_{i=0}^{n} G_{i}\right]^{T}=\sum_{i=0}^{n} E\left[G_{i}\right]^{T} \stackrel{(7)}{=} \sum_{i=0}^{n} E\left[G_{0}\right]^{T} \cdot\left(M_{C}\right)^{n}
$$

It is possible to write the resulting sum as the closed formula:

$$
E\left[P_{n}\right]^{T}=E\left[G_{0}\right]^{T} \cdot\left(\frac{I-\left(M_{C}\right)^{n+1}}{I-M_{C}}\right)
$$

where $I$ represents the identity matrix of the appropriate size. Note that equation (9) only holds when $\left(I-M_{C}\right)$ is nonsingular, however, this is the usual case. When $\left(I-M_{C}\right)$ is singular, we resort to using equation (8) instead. Without losing generality, and for simplicity, we consider equations (8) and (9) as interchangeable. They are the general formulas for the Galton-Watson multi-type branching processes.

Then, to predict the distribution of our Tree' data type example, we proceed to build its mean matrix $M_{C}$. For instance, the mean number of Leafs generated by a $\mathrm{Node}_{A}$ is:

$$
\begin{aligned}
m_{\text {Node }_{A}, \text { Leaf }} & =\underbrace{1 \cdot p_{\text {Leaf }} \cdot p_{\text {Node }_{A}}+1 \cdot p_{\text {Leaf }} \cdot p_{\text {Node }_{B}}}_{\text {One Leaf as left-subtree }} \\
& +\underbrace{1 \cdot p_{\text {Node }_{A}} \cdot p_{\text {Leaf }}+1 \cdot p_{N_{\text {Lode }}} \cdot p_{\text {Leaf }}}_{\text {One Leaf as right-subtree }} \\
& +\underbrace{2 \cdot p_{\text {Leaf }} \cdot p_{\text {Leaf }}}_{\text {Leaf } \text { as left- and right-subtree }} \\
& =2 \cdot p_{\text {Leaf }}
\end{aligned}
$$

The rest of $M_{C}$ can be similarly computed, obtaining:

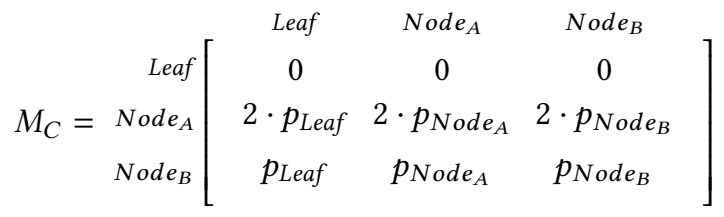

Note that the first row, corresponding to the Leaf constructor, is filled with zeros. This is because Leaf is a terminal constructor, i.e., it cannot generate further subterms of any kind. ${ }^{5}$

With the mean matrix in place, we define $E\left[G_{0}\right]$ (the initial vector of mean probabilities) as ( $\left.p_{\text {Leaf }}, p_{\mathrm{Node}_{A}}, p_{\mathrm{Node}_{B}}\right)$. By applying (9) with $E\left[G_{0}\right]$ and $M_{C}$, we can predict the expected number of generated non-terminal Node $_{A}$ constructors (and analogously $N_{o d e}$ ) with a size parameter $n$ as follows:

$E\left[\operatorname{Node}_{\mathrm{A}}\right]=\left(E\left[P_{n-1}\right]^{T}\right) \cdot \operatorname{Node}_{\mathrm{A}}=\left(E\left[G_{0}\right]^{T} \cdot\left(\frac{I-\left(M_{C}\right)^{n}}{I-M_{C}}\right)\right) \cdot$ Node $_{\mathrm{A}}$

Function (_).C simply projects the value corresponding to constructor $C$ from the population vector. It is very important to note that the sum only includes the population up to level $(n-1)$. This choice comes from the fact that our QuickCheck generator can choose between only terminal constructors at the last generation level (recall that gen 0 generates only Leafs in Figure 5). As an example, if we assign our generation probabilities for Tree' as $p_{\text {Leaf }} \mapsto 0.2, p_{\text {Node }_{A}} \mapsto 0.5$ and $p_{\text {Node }_{B}} \mapsto 0.3$, then the formula predicts that our QuickCheck generator with a size parameter of 10 will generate on average 21.322 Node $_{A} \mathrm{~s}$ and 12.813 Node $_{B} \mathrm{~s}$. This result can easily be verified by sampling a large number of values with a generation size of 10 , and then averaging the number of generated Node $_{A} \mathrm{~s}$ and Node $_{B} \mathrm{~s}$ across the generated values.

In this section, we obtain a prediction of the expected number of non-terminal constructors generated by DRAGEN generators. To predict terminal constructors, however, requires a special treatment as discussed in the next section.

\footnotetext{
${ }^{5}$ The careful reader may notice that there is a pattern in the mean matrix if inspected together with the definition of Tree'. We prove in Section 6 that each $m_{i j}$ can be automatically calculated by simply exploiting type information.
} 


\section{Terminal constructors}

In this section we introduce the special treatment required to predict the generated distribution of terminal constructors, i.e. constructors with no recursive arguments.

Consider the generator in Figure 5. It generates terminal constructors in two situations, i.e., in the definition of gen 0 and gen $n$. In other words, the random process introduced by our generators can be considered to be composed of two independent parts when it comes to terminal constructors-refer to the supplementary material for a graphical interpretation. In principle, the number of terminal constructors generated by the stochastic process described in gen $n$ is captured by the multi-type branching process formulas. However, to predict the expected number of terminal constructors generated by exercising gen 0 , we need to separately consider a random process that only generates terminal constructors in order to terminate. For this purpose, and assuming a maximum generation depth $n$, we need to calculate the number of terminal constructors required to stop the generation process at the recursive arguments of each non-terminal constructor at level $(n-1)$. In our Tree' example, this corresponds to two Leafs for every Node $A$ and one Leaf for every Node $_{B}$ constructor at level $(n-1)$.

Since both random processes are independent, to predict the overall expected number of terminal constructors, we can simply add the expected number of terminal constructors generated in each one of them. Recalling our previous example, we obtain the following formula for Tree' terminals as follows:

$$
\begin{aligned}
E[\text { Leaf }]=\underbrace{\left(E\left[P_{n-1}\right]^{T}\right) \cdot \text { Leaf }}_{\text {branching process }} & +\underbrace{2 \cdot\left(E\left[G_{n-1}\right]^{T}\right) \cdot \text { Node }_{\mathrm{A}}}_{\text {case }\left(\text { Node }_{A} \text { Leaf Leaf }\right)} \\
& +\underbrace{1 \cdot\left(E\left[G_{n-1}\right]^{T}\right) \cdot \text { Node }_{B}}_{\text {case }\left(\text { Node }_{B} \text { Leaf }\right)}
\end{aligned}
$$

The formula counts the Leafs generated by the multi-type branching process up to level $(n-1)$ and adds the expected number of Leafs generated at the last level.

Although we can now predict the expected number of generated Tree' constructors regardless of whether they are terminal or not, this approach only works for data types with a single terminal constructor. If we have a data type with multiple terminal constructors, we have to consider the probabilities

$$
\begin{aligned}
& \text { instance Arbitrary Tree" where } \\
& \text { arbitrary }=\text { sized gen } \text { where } \\
& \text { gen } 0=\text { chooseWith } \\
& {\left[\left(p_{\text {Leaf } A}^{*}, \text { pure Leaf } f_{A}\right),\left(p_{\text {Leaf } B}^{*}, \text { pure Leaf } f_{B}\right)\right]} \\
& \text { gen } n=\text { chooseWith } \\
& {\left[\left(p_{\text {Leaf } A} \text {, pure Leaf } f_{A}\right),\left(p_{\text {Leaf } B}, \text { pure Leaf } f_{B}\right)\right.} \\
& ,\left(p_{\text {Node }_{A}, \text { Node }_{A}\langle \$\rangle \text { gen }}(n-1)\langle *\rangle \text { gen }(n-1)\right) \\
& \left.,\left(p_{\text {NodeB }}, \operatorname{Node}_{B}\langle \$\rangle \operatorname{gen}(n-1)\right)\right]
\end{aligned}
$$

Figure 7. Derived generator for Tree" of choosing each one of them when filling the recursive arguments of non-terminal constructors at the previous level. For instance, consider the following ADT:

data Tree" $=$ Leaf $_{A} \mid$ Leaf $_{B} \mid$ Node $_{A}$ Tree" $^{\prime \prime}$ Tree" $^{\prime \prime} \mid$ Node $_{B}$ Tree" $^{\prime \prime}$

Figure 7 shows the corresponding DRAGEN generator for Tree". Note there are two sets of probabilities to choose terminal nodes, one for each random process. The $p_{\text {Leaf } A}^{*}$ and $p_{\text {Leaf } B}^{*}$ probabilities are used to choose between terminal constructors at the last generation level. These probabilities preserve the same proportion as their non-starred versions, i.e., they are normalized to form a probability distribution:

$$
p_{\text {Leaf }_{A}}^{*}=\frac{p_{\text {Leaf }_{A}}}{p_{\text {Leaf }_{A}}+p_{\text {Leaf }_{B}}} \quad p_{\text {Leaf }_{B}}^{*}=\frac{p_{\text {Leaf }_{B}}}{p_{\text {Leaf }_{A}}+p_{\text {Leaf }_{B}}}
$$

In this manner, we can use the same generation probabilities for terminal constructors in both random processes-therefore reducing the complexity of our prediction engine implementation (described in Section 7).

To compute the overall expected number of terminals, we need to predict the expected number of terminal constructors at the last generation level which could be descendants of non-terminal constructors at level $(n-1)$. More precisely:

$$
\begin{aligned}
& E\left[\text { Leaf }_{A}\right]=\underbrace{\left(E\left[P_{n-1}\right]^{T}\right) \cdot \text { Leaf }_{\mathrm{A}}}_{\text {branching process }}+\underbrace{2 \cdot p_{\text {Leaf }_{A}}^{*} \cdot\left(E\left[G_{n-1}\right]^{T}\right) \cdot \text { Node }_{\mathrm{A}}}_{\text {expected leaves to fill Node } \text { S }_{A}} \\
& +\underbrace{1 \cdot p_{\text {Leaf }_{A}}^{*} \cdot\left(E\left[G_{n-1}\right]^{T}\right) \cdot \text { Node }_{\mathrm{B}}}_{\text {expected leaves to fill } \text { Node }_{B} S}
\end{aligned}
$$

where the case of $E\left[\operatorname{Leaf}_{B}\right]$ follows analogously.

\section{Mutually-recursive and composite ADTs}

In this section, we introduce some extensions to our model that allow us to derive DRAGEN generators for data types found in existing off-the-shelf Haskell libraries. We start by showing how multi-type branching processes naturally extend to mutually-recursive ADTs. Consider the mutually recursive ADTs $T_{1}$ and $T_{2}$ with their automatically derived generators shown in Figure 8. Note the use of the QuickCheck's function resize::Int $\rightarrow$ Gen $a \rightarrow$ Gen $a$, which resets the generation size of a given generator to a new value. We use it to decrement the generation size at the recursive calls of arbitrary that generate subterms of a mutually recursive data type.

The key observation is that we can ignore that $A, B, C$ and $D$ are constructors belonging to different data types and just consider each of them as a kind of offspring on its own. Figure 9 visualizes the possible offspring generated by the non-terminal constructor $B$ (belonging to $T_{1}$ ) with the corresponding probabilities as labeled edges. Following the figure, we obtain the expected number of $D$ s generated by $B$ constructors as follows:

$$
m_{B D}=1 \cdot p_{A} \cdot p_{D}+1 \cdot p_{B} \cdot p_{D}=p_{D} \cdot\left(p_{A}+p_{B}\right)=p_{D}
$$




$$
\begin{aligned}
& \text { data } T_{1}=A \mid B T_{1} T_{2} \\
& \text { data } T_{2}=C \mid D T_{1} \\
& \text { instance Arbitrary } T_{1} \text { where } \\
& \text { arbitrary }=\text { sized gen where } \\
& \quad \text { gen } 0=\text { pure } A \\
& \quad \text { gen } n=\text { chooseWith } \\
& \quad\left[\left(p_{A}, \text { pure } A\right)\right. \\
& \left.\quad,\left(p_{B}, B\langle \$\rangle \text { gen }(n-1)\langle *\rangle \text { resize }(n-1) \text { arbitrary }\right)\right] \\
& \text { instance Arbitrary } T_{2} \text { where } \\
& \text { arbitrary }=\text { sized gen where } \\
& \quad \text { gen } 0=\text { pure } C \\
& \text { gen } n=\text { chooseWith } \\
& \quad\left[\left(p_{C}, \text { pure } C\right),\left(p_{D}, D\langle \$\rangle \text { resize }(n-1) \text { arbitrary }\right)\right]
\end{aligned}
$$

Figure 8. Mutually recursive types $T_{1}$ and $T_{2}$ and their DRAGEN generators.

Doing similar calculations, we obtain the mean matrix $M_{C}$ for $A, B, C$, and $D$ as follows:

$$
M_{C}={ }_{C}{ }_{C}\left[\begin{array}{cc:cc}
A & B & C & D \\
0 & 0 & 0 & 0 \\
p_{A} & p_{B} & p_{C} & p_{D} \\
\hdashline 0 & 0 & 0 & 0 \\
p_{A} & p_{B} & 0 & 0
\end{array}\right]
$$

We define the mean of the initial generation as $E\left[G_{0}\right]=$ $\left(p_{A}, p_{B}, 0,0\right)$-we assing $p_{C}=p_{D}=0$ since we choose to start by generating a value of type $T_{1}$. With $M_{C}$ and $E\left[G_{0}\right]$ in place, we can apply the equations explained through Section 4 to predict the expected number of $A, B, C$ and $D$ constructors.

While this approach works, it completely ignores the types $T_{1}$ and $T_{2}$ when calculating $M_{C}$ ! For a large set of mutuallyrecursive data types involving a large number of constructors, handling $M_{C}$ like this results in a high computational cost. We show next how we cannot only shrink this mean matrix of constructors but also compute it automatically by making use of data type definitions.

Mean matrix of types If we analyze the mean matrices of Tree $e^{\prime}$ (11) and the mutually-recursive types $T_{1}$ and $T_{2}$ (12), it seems that determining the expected number of offspring generated by a non-terminal constructor requires us to count

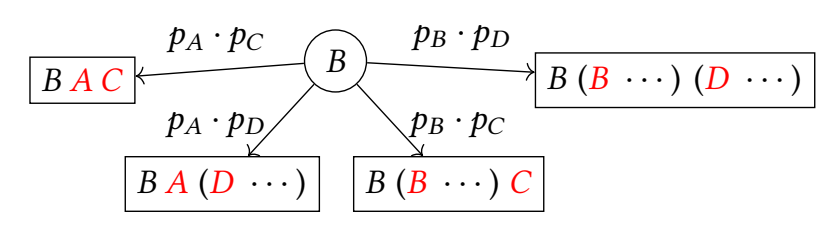

Figure 9. Possible offspring of constructor $B$. the number of occurrences in the ADT which the offspring belongs to. For instance, $m_{\text {NodeA, Leaf }}$ is $2 \cdot p_{\text {Leaf }}(10)$, where 2 is the number of occurrences of Tree' in the declaration of Node $_{A}$. Similarly, $m_{B D}$ is $1 \cdot p_{D}$, where 1 is the number of occurrences of $T_{2}$ in the declaration of $B$. This observation means that instead of dealing with constructors, we could directly deal with types!

We can think about a branching process as generating "place holders" for constructors, where place holders can only be populated by constructors of a certain type.

Figure 10 illustrates offspring as types for the definitions $T_{1}, T_{2}$, and Tree'. A place holder of type $T_{1}$ can generate a place holder for type $T_{1}$ and a place holder for type $T_{2}$. A place holder of type $T_{2}$ can generate a place holder of type $T_{1}$. A place holder of type Tree $e^{\prime}$ can generate two place holders of type Tree' when generating $\operatorname{Node}_{A}$, one place holder when generating $N_{0 d e_{B}}$, or zero place holders when generating a Leaf (this last case is not shown in the figure since it is void). With these considerations, the mean matrices of types for Tree', written $M_{\text {Tree }}$; and types $T_{1}$ and $T_{2}$, written $M_{T_{1} T_{2}}$ are defined as follows:

$$
M_{\text {Tree }}=\text { Tree }^{\text {Tree }^{\prime}}\left[2 \cdot p_{\text {NodeA }}+p_{\text {Node } B}\right] \quad M_{T_{1} T_{2}}={ }_{T 2}^{T 1}\left[\begin{array}{cc}
T 1 & T 2 \\
p_{B} & p_{B} \\
p_{D} & 0
\end{array}\right]
$$

Note how $M_{\text {Tree }}$ shows that the mean matrices of types might reduce a multi-type branching process to a simple-type one.

Having the type matrix in place, we can use the following equation (formally stated and proved in the supplementary material) to soundly predict the expected number of constructors of a given set of (possibly) mutually recursive types:

$$
\left(E\left[G_{n}^{C}\right]\right) \cdot C_{i}^{t}=\left(E\left[G_{n}^{T}\right]\right) \cdot T_{t} \cdot p_{C_{i}^{t}}
$$

Where $G_{n}^{C}$ and $G_{n}^{T}$ denotes the $n$ th-generations of constructors and type place holders respectively. $C_{i}^{t}$ represents the $i$ th-constructor of the type $T_{t}$. The equation establishes that, the expected number of constructors $C_{i}^{t}$ at generation $n$ consists of the expected number of type place holders of its type (i.e., $T_{t}$ ) at generation $n$ times the probability of generating that constructor. This equation allows us to simplify many of our calculations above by simply using the mean matrix for types instead of the mean matrix for constructors.
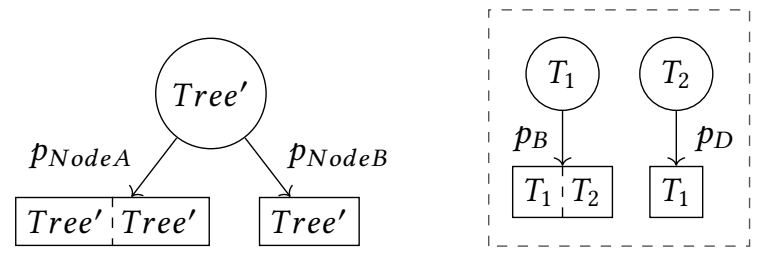

Figure 10. Offspring as types 


\subsection{Composite types}

In this subsection, we extend our approach in a modular manner to deal with composite ADTs, i.e., ADTs which use already defined types in their constructors' arguments and which are not involved in the branching process. We start by considering the ADT Tree modified to carry booleans at the leaves:

data Tree $=$ Lea $_{A}$ Bool $\mid$ Lea $_{B}$ Bool Bool $\mid \cdots$

Where $\cdots$ denotes the constructors that remain unmodified. To predict the expected number of True (and analogously of False) constructors, we calculate the multi-type branching process for Tree and multiply each expected number of leaves by the number of arguments of type Bool present in each one:

$$
E[\text { True }]=p_{\text {True }} \cdot(\underbrace{1 \cdot E\left[\text { Leaf }_{A}\right]}_{\text {case } \text { Leaf }_{A}}+\underbrace{\left.2 \cdot \text { E Leaf }_{B}\right]}_{\text {case } \text { Leaf }_{B}})
$$

In this case, Bool is a ground type like Int, Float, etc. Predictions become more interesting when considering richer composite types involving, for instance, instantiations of polymorphic types. To illustrate this point, consider a modified version of Tree where $L e a f_{A}$ now carries a value of type Maybe Bool:

data Tree $=$ Lea $_{A}($ Maybe Bool $) \mid$ Lea $_{B}$ Bool Bool $\mid \cdots$

In order to calculate the expected number of Trues, now we need to consider the cases that a value of type Maybe Bool actually carries a boolean value, i.e., when a fust constructor gets generated:

$$
E[\text { True }]=p_{\text {True }} \cdot\left(1 \cdot E\left[\text { Leaf }_{A}\right] \cdot p_{\text {Just }}+2 \cdot E\left[\text { Lea }_{B}\right]\right)
$$

In the general case, for constructor arguments utilizing other ADTs, it is necessary to know the chain of constructors required to generate "foreign" valuesin our example, a True value gets generated if a $\operatorname{Leaf}_{A}$ gets generated with a fust constructor "in between." To obtain such information, we

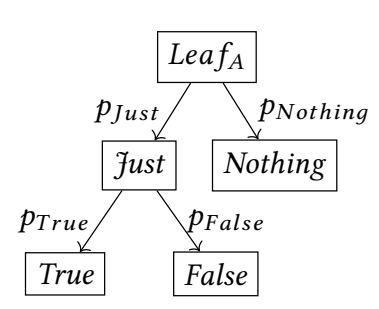

Figure 11. Constructor dependency graph. create of a constructor dependency graph (CDG), that is, a directed graph where each node represents a constructor and each edge represents its dependency. Each edge is labeled with its corresponding generation probability. Figure 11 shows the CDG for Tree starting from the Lea $f_{A}$ constructor. Having this graph together with the application of the multi-type branching process, we can predict the expected number of constructors belonging to external ADTs. It is enough to multiply the probabilities at each edge of the path between every constructor involved in the branching process and the desired external constructor.

The extensions described so far enable our tool (presented in the next section) to make predictions about QuickCheck generators for ADTs defined in many existing Haskell libraries.

\section{Implementation}

DRAGEN is a tool chain written in Haskell that implements the multi-type branching processes (Section 4 and 5) and its extensions (Section 6) together with a distribution optimizer, which calibrates the probabilities involved in generators to fit developers' demands. DRAGEN synthesizes generators by calling the Template Haskell function dragenArbitrary:: Name $\rightarrow$ Size $\rightarrow$ CostFunction $\rightarrow Q$ [Dec], where developers indicate the target ADT for which they want to obtain a QuickCheck generator; the desired generation size, needed by our prediction mechanism in order to calculate the distribution at the last generation level; and a cost function encoding the desired generation distribution.

The design decision to use a probability optimizer rather than search for an analytical solution is driven by two important aspects of the problem we aim to solve. Firstly, the computational cost of exactly solving a non-linear system of equations (such as those arising from branching processes) can be prohibitively high when dealing with a large number of constructors, thus a large number of unknowns to be solved for. Secondly, the existence of such exact solutions is not guaranteed due to the implicit invariants the data types under consideration might have. In such cases, we believe it is much more useful to construct a distribution that approximates the user's goal, than to abort the entire compilation process. We give an example of this approximate solution finding behavior later in this section.

\subsection{Cost functions}

The optimization process is guided by a user-provided cost function. In our setting, a cost function assigns a real number (a cost) to the combination of a generation size (chosen by the user) and a mapping from constructors to probabilities:

type CostFunction $=$ Size $\rightarrow$ ProbMap $\rightarrow$ Double

Type ProbMap encodes the mapping from constructor names to real numbers. Our optimization algorithm works by generating several ProbMap candidates that are evaluated through the provided cost function in order to choose the most suitable one. Cost functions are expected to return a smaller positive number as the predicted distribution obtained from its parameters gets closer to a certain target distribution, which depends on what property that particular cost function is intended to encode. Then, the optimizator simply finds the best ProbMap by minimizing the provided cost function.

Currently, our tool provides a basic set of cost functions to easily describe the expected distribution of the derived generator. For instance, uniform :: CostFunction encodes constuctorwise uniform generation, an interesting property that naturally arises from our generation process formalization. It guides the optimization process to a generation distribution that minimizes the difference between the expected number of each generated constructor and the generation size. 
Moreover, the user can restrict the generation distribution to a certain subset of constructors using the cost functions only :: [Name] $\rightarrow$ CostFunction and without $::[$ Name $] \rightarrow$ CostFunction to describe these restrictions. In this case, the whitelisted constructors are then generated following the uniform behavior. Similarly, if the branching process involves mutually recursive data types, the user could restrict the generation to a certain subset of data types by using the functions onlyTypes and withoutTypes. Additionally, when the user wants to generate constructors according to certain proportions, weighted :: $[($ Name, Int $)] \rightarrow$ CostFunction allows to encode this property, e.g. three times more $L e a f_{A}$ 's than $L e a f_{B}$ 's.

Table 1 shows the number of expected and observed constructors of different Tree generators obtained by using different cost functions. The observed expectations were calculated averaging the number of constructors across 100000 generated values. Firstly, note how the generated distributions are soundly predicted by our tool. In our tests, the small differences between predictions and actual values dissapear as we increase the number of generated values. As for the cost functions' behavior, there are some interesting aspects to note. For instance, in the uniform case the optimizer cannot do anything to break the implicit invariant of the data type: every binary tree with $n$ nodes has $n+1$ leaves. Instead, it converges to a solution that "approximates" a uniform distribution around the generation size parameter. We believe this is desirable behavior, to find an approximate solution when certain invariants prevent the optimization process from finding an exact solution. This way the user does not have to be aware of the possible invariants that the target data type may have, obtaining a solution that is good enough for most purposes. On the other hand, notice that in the weighted case at the second row of Table 1 , the expected number of generated Nodes is considerably large. This constructor is not listed in the proportions list, hence the optimizer can freely adjust its probability to satisfy the proportions specified for the leaves.

\subsection{Derivation Process}

DRAGEN's derivation process starts at compile-time with a type reification stage that extracts information about the structure of the types under consideration. It follows an intermediate stage composed of the optimizer for probabilities used in generators, which is guided by our multi-type branching process model, parametrized on the cost function provided. This optimizer is based on a standard local-search optimization algorithm that recursively chooses the best mapping from constructors to probabilities in the current neighborhood. Neighbors are ProbMaps, determined by individually varying the probabilities for each constructor with a predetermined $\Delta$. Then, to determine the "best" probabilities, the local-search applies our prediction mechanishm to the immediate neighbors that have not yet been visited by evaluating the cost function to select the most suitable next candidate. This process continues until a local minimum is reached when there are no new neighbors to evaluate, or if each step improvement is lower than a minimum predetermined $\varepsilon$.

The final stage synthesizes a Arbitrary type-class instance for the target data types using the optimized generation probabilities. For this stage, we extend some functionality present in MegaDeTH in order to derive generators parametrized by our previously optimized probabilities. Refer to the supplementary material for further details on the cost functions and algorithms addressed by this section.

\section{Case Studies}

We start by comparing the generators for the ADT Tree derived by MegaDeTH and Feat, presented in Section 2, with the corresponding generator derived by DRAGEN using a uniform cost function. We used a generation size of 10 both for MegaDeTH and DRAGEN, and a generation size of 400 for Feat-that is, Feat will generate test cases of maximum 400 constructors, since this is the maximum number of constructors generated by our tool using the generation size cited above. Figure 12 shows the differences between the complexity of the generated values in terms of the number of constructors. As shown in Figure 3, generators derived by MegaDeTH and Feat produce very narrow distributions, being unable to generate a diverse variety of values of different sizes. In contrast, the DRAGEN optimized generator provides a much wider distribution, i.e., from smaller to bigger values.

It is likely that the richer the values generated, the better the chances of covering more code, and thus of finding more bugs. The next case studies provide evidence in that direction.

Although DRAGEN can be used to test Haskell code, we follow the same philosophy as QuickFuzz, targeting three complex and widely used external programs to evaluate how well our derived generators behave. These applications are GNU bash 4.4-a widely used Unix shell, GNU CLISP 2.49-the GNU Common Lisp compiler, and giffix-a small test utility from the GIFLIB 5.1 library focused on reading and writing Gif images. It is worth noticing that these applications are not written in Haskell. Nevertheless, there are Haskell libraries designed to inter-operate with them: language-bash, atto-lisp, and fuicyPixels, respectively. These libraries provide ADT definitions

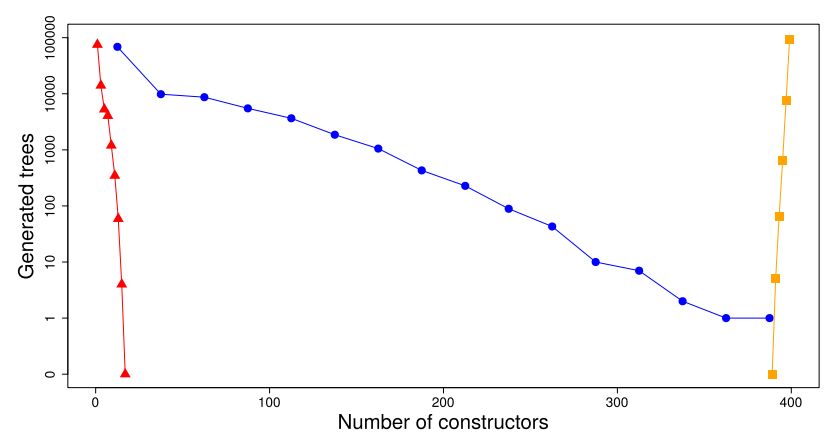

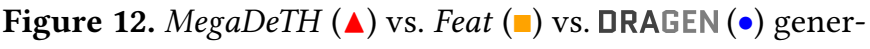
ated distributions for type Tree. 
Table 1. Predicted and actual distributions for Tree generators using different cost functions.

\begin{tabular}{|c|c|c|c|c|c|c|c|c|}
\hline \multirow[t]{2}{*}{ Cost Function } & \multicolumn{4}{|c|}{ Predicted Expectation } & \multicolumn{4}{|c|}{ Observed Expectation } \\
\hline & $\operatorname{Leaf}_{A}$ & $\operatorname{Leaf}_{B}$ & Leaf $f_{C}$ & Node & $\operatorname{Leaf}_{A}$ & $\operatorname{Leaf}_{B}$ & Leaf $f_{C}$ & Node \\
\hline uniform & 5.26 & 5.26 & 5.21 & 14.73 & 5.27 & 5.26 & 5.21 & 14.74 \\
\hline weighted $\left[\left({ }^{\prime}\right.\right.$ Lea $\left._{A}, 3\right),\left({ }^{\prime} \operatorname{Leaf}_{B}, 1\right),\left({ }^{\prime}\right.$ Lea $\left.\left._{C}, 1\right)\right]$ & 30.07 & 9.76 & 10.15 & 48.96 & 30.06 & 9.75 & 10.16 & 48.98 \\
\hline weighted $\left[\left({ }^{\prime}\right.\right.$ Leaf $\left.f_{A}, 1\right),\left({ }^{\prime}\right.$ Node, 3$\left.)\right]$ & 10.07 & 3.15 & 17.57 & 29.80 & 10.08 & 3.15 & 17.58 & 29.82 \\
\hline only $\left[{ }^{\prime} L e a f_{A},{ }^{\prime} N o d e\right]$ & 10.41 & 0 & 0 & 9.41 & 10.43 & 0 & 0 & 9.43 \\
\hline without $\left[{ }^{\prime} L e a f_{C}\right]$ & 6.95 & 6.95 & 0 & 12.91 & 6.93 & 6.92 & 0 & 12.86 \\
\hline
\end{tabular}

which we used to synthesize DRAGEN generators for the inputs of the aforementioned applications. Moreover, they also come with serialization functions that allow us to transform the randomly generated Haskell values into the actual test files that we used to test each external program. The case studies contain mutually recursive and composite ADTs with a wide number of constructors (e.g., GNU bash spans 31 different ADTs and 136 different constuctors)-refer to the supplementary material for a rough estimation of the scale of such data types and the data types involved with them.

For our experiments, we use the coverage measure known as execution path employed by American Fuzzy Lop (AFL) [20]-a well known fuzzer. It was chosen in this work since it is also used in the work by Grieco et al. [14] to compare MegaDeTH with other techniques. The process consists of the instrumentation of the binaries under test, making them able to return the path in the code taken by each execution. Then, we use AFL to count how many different executions are triggered by a set of randomly generated files-also known as a corpus. In this evaluation, we compare how different QuickCheck generators, derived using MegaDeTH and using our approach, result in different code coverage when testing external programs, as a function of the size of a set of independently, randomly generated corpora. We have not been able to automatically derive such generators using Feat, since it does not work with some Haskell extensions used in the bridging libraries.

We generated each corpus using the same ADTs and generation sizes for each derivation mechanism. We used a generation size of 10 for CLISP and bash files, and a size of 5 for Gif files. For DRAGEN, we used uniform cost functions to reduce any external bias. In this manner, any observed difference in the code coverage triggered by the corpora generated using each derivation mechanism is entirely caused by the optimization stage that our predictive approach performs, which does not represent an extra effort for the programmer. Moreover, we repeat each experiment 30 times using independently generated corpora for each combination of derivation mechanism and corpus size.

Figure 13 compares the mean number of different execution paths triggered by each pair of generators and corpus sizes, with error bars indicating $95 \%$ confidence intervals of the mean. It is easy to see how the DRAGEN generators synthesize test cases capable of triggering a much larger number of different execution paths in comparison to MegaDeTH ones. Our results indicate average increases approximately between $35 \%$ and $41 \%$ with an standard error close to $0.35 \%$ in the number of different execution paths triggered in the programs under test.

An attentive reader might remember that MegaDeTH tends to derive generators which produce very small test cases. If we consider that small test cases should take less time (on average) to be tested, is fair to think there is a trade-off between being able to test a bigger number of smaller test cases or a smaller number of bigger ones having the same time available. However, when testing external software like in our experiments, it is important to consider the time overhead introduced by the operating system. In this scenario, it is much more preferable to test interesting values over smaller ones. In our tests, size differences between the generated values of each tool does do not result in significant differences in the runtimes required to test each corpora-refer to the supplementary material for further details. A user is most likely to get better results by using our tool instead of MegaDeTH, with virtually the same effort.

We also remark that, if we run sufficiently many tests, then the expected code coverage will tend towards $100 \%$ of the reachable code in both cases. However, in practice, our approach is more likely to achieve higher code coverage for the same number of test cases.

\section{Related Work}

Fuzzers are tools to tests programs against randomly generated unexpected inputs. QuickFuzz [13,14] is a tool that synthesizes data with rich structure, that is, well-typed files which can be used as initial "seeds" for state-of-the-art fuzzers-a work flow which discovered many unknown vulnerabilities. Our work could help to improve the variation of the generated initial seeds, by varying the distribution of QuickFuzz generators-an interesting direction for future work.

SmallCheck [27] provides a framework to exhaustively test data sets up to a certain (small) size. The authors also propose a variation called Lazy SmallCheck, which avoids the generation of multiple variants which are passed to the test, but not actually used.

QuickCheck has been used to generate well-typed lambda terms in order to test compilers [25]. Recently, Midtgaard et al. extend such a technique to test compilers for impure programming languages [22]. 

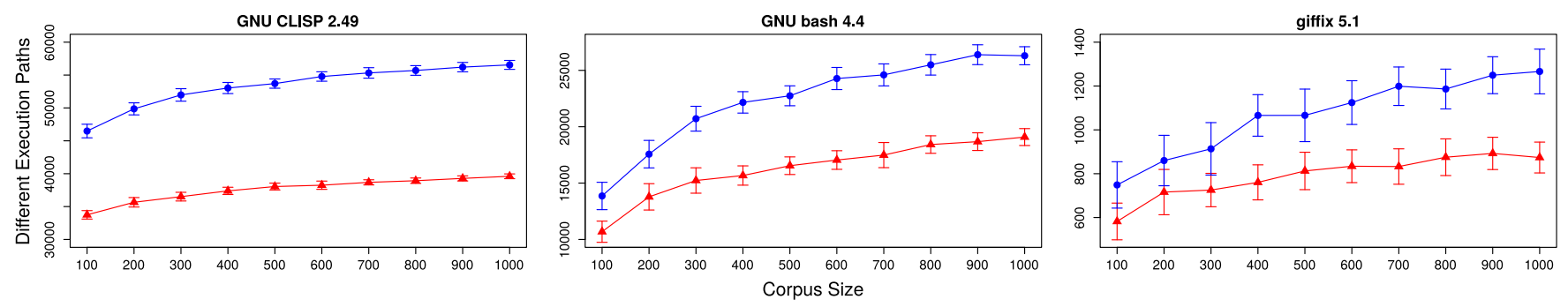

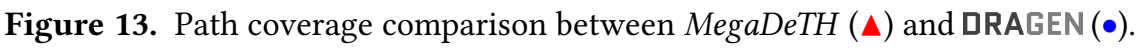

Luck [18] is a domain specific language for writing testing properties and QuickCheck generators at the same time. We see Luck's approach as orthogonal to ours, which is mostly intended to be used when we do not know any specific property of the system under test, although we consider that borrowing some functionality from Luck into DRAGEN is an interesting path for future work.

Recently, Lampropoulos et al. propose a framework to automatically derive random generators for a large subclass of Coqs' inductively defined relations [19]. This derivation process also provides proof terms certifying that each derived generator is sound and complete with respect to the inductive relation it was derived from.

Boltzmann models [10] are a general approach to randomly generating combinatorial structures such as trees and graphsalso extended to work with closed simply-typed lambda terms [4]. By implementing a Boltzmann sampler, it is possible to obtain a random generator built around such models which uniformly generates values of a target size with a certain size tolerance. However, this approach has practical limitations Firstly, the framework is not expressive enough to represent complex constrained data structures, e.g red-black trees. Secondly, Boltzmann samplers give the user no control over the distribution of generated values besides ensuring size-uniform generation. They work well in theory but further work is required to apply them to complex structures [26]. Conversely, DRAGEN provides a simple mechanism to predict and tune the overall distribution of constructors analytically at compiletime, using statically known type information, and requiring no runtime reinforcements to ensure the predicted distributions. Future work will explore the connections between branching processes and Boltzmann models.

Similarly to our work, Feldt and Poulding propose GödelTest [12], a search-based framework for generating biased data. It relies on non-determinism to generate a wide range of data structures, along with metaheuristic search to optimize the parameters governing the desired biases in the generated data. Rather than using metaheuristic search, our approach employs a completely analytical process to predict the generation distribution at each optimization step. A strength of the GödelTest approach is that it can optimize the probability parameters even when there is no specific target distribution over the constructors-this allows exploiting software behavior under test to guide the parameter optimization.

The efficiency of random testing is improved if the generated inputs are evenly spread across the input domain [5]. This is the main idea of Adaptive Random Testing (ART) [6]. However, this work only covers the particular case of testing programs with numerical inputs and it has also been argued that adaptive random testing has inherent inefficiencies compared to random testing [1]. This strategy is later extended in [7] for object-oriented programs. These approaches present no analysis of the distribution obtained by the heuristics used, therefore we see them as orthogonal work to ours.

\section{Final Remarks}

We discover an interplay between the stochastic theory of branching processes and algebraic data types structures. This connection enables us to describe a solid mathematical foundation to capture the behavior of our derived QuickCheck generators. Based on our formulas, we implement a heuristic to automatically adjust the expected number of constructors being generated as a way to control generation distributions.

One holy grail in testing is the generation of structured data which fulfills certain invariants. We believe that our work could be used to enforce some invariants on data "up to some degree." For instance, by inspecting programs' source code, we could extract the pattern-matching patterns from programs (e.g., (Cons $($ Cons $x)))$ and derive generators which ensure that such patterns get exercised a certain amount of times (on average)-intriguing thoughts to drive our future work.

\section{Acknowledgments}

We would like to thank Michał Pałka, Nick Smallbone, Martin Ceresa and Gustavo Grieco for comments on an early draft. This work was funded by the Swedish Foundation for Strategic Research (SSF) under the project Octopi (Ref. RIT17-0023) and WebSec (Ref. RIT17-0011) as well as the Swedish research agency Vetenskapsrådet.

\section{References}

[1] A. Arcuri and L. Briand. 2011. Adaptive Random Testing: An Illusion of Effectiveness?. In Proc. of the International Symposium on Software Testing and Analysis (ISSTA '11). ACM.

[2] B. Arkin, S. Stender, and G. McGraw. 2005. Software penetration testing. IEEE Security Privacy (2005). 
[3] T. Arts, J. Hughes, U. Norell, and H. Svensson. 2015. Testing AUTOSAR software with QuickCheck. In In Proc. of IEEE International Conference on Software Testing, Verification and Validation, ICST Workshops.

[4] M. Bendkowski, K. Grygiel, and P. Tarau. 2017. Boltzmann Samplers for Closed Simply-Typed Lambda Terms. In In Proc. of International Symposium on Practical Aspects of Declarative Languages. ACM.

[5] F.T. Chan, T.Y. Chen, I.K. Mak, and Y.T. Yu. 1996. Proportional sampling strategy: guidelines for software testing practitioners. Information and Software Technology 38, 12 (1996), 775 - 782.

[6] T. Y. Chen, H. Leung, and I. K. Mak. 2005. Adaptive Random Testing. In Advances in Computer Science - ASIAN 2004. Higher-Level Decision Making, Michael J. Maher (Ed.). Springer Berlin Heidelberg.

[7] I. Ciupa, A. Leitner, M. Oriol, and B. Meyer. 2008. ARTOO: adaptive random testing for object-oriented software. In Proc. of International Conference on Software Engineering. ACM/IEEE.

[8] K. Claessen, J. Duregård, and M. H. Palka. 2014. Generating Constrained Random Data with Uniform Distribution. In Proc. of the Functional and Logic Programming FLOPS.

[9] K. Claessen and J. Hughes. 2000. QuickCheck: A Lightweight Tool for Random Testing of Haskell Programs. In Proc. of the ACM SIGPLAN International Conference on Functional Programming (ICFP).

[10] P. Duchon, P. Flajolet, G. Louchard, and G. Schaeffer. 2004. Boltzmann Samplers for the Random Generation of Combinatorial Structures. Com binatorics, Probability and Computing. 13 (2004).

[11] J. Duregård, P. Jansson, and M. Wang. 2012. Feat: Functional enumeration of algebraic types. In Proc. of the ACM SIGPLAN Symposium on Haskell.

[12] R. Feldt and S. Poulding. 2013. Finding test data with specific properties via metaheuristic search. In Proc. of International Symp. on Software Reliability Engineering (ISSRE). IEEE.

[13] G. Grieco, M. Ceresa, and P. Buiras. 2016. QuickFuzz: An automatic random fuzzer for common file formats. In Proc. of the International Symposium on Haskell. ACM.

[14] G. Grieco, M. Ceresa, A. Mista, and P. Buiras. 2017. QuickFuzz testing for fun and profit. Journal of Systems and Software 134, Supp. C (2017).

[15] P. Haccou, P. Jagers, and V. Vatutin. 2005. Branching processes. Variation, growth, and extinction of populations. Cambridge University Press.

[16] J. Hughes, C. Pierce B, T. Arts, and U. Norell. 2016. Mysteries of DropBox: Property-Based Testing of a Distributed Synchronization Service. In Proc. of the Int. Conference on Software Testing, Verification and Validation, ICST.

[17] J. Hughes, U. Norell, N. Smallbone, and T. Arts. 2016. Find more bugs with QuickCheck!. In Proc. of the International Workshop on Automation of Software Test, AST@ICSE.

[18] L. Lampropoulos, D. Gallois-Wong, C. Hritcu, J. Hughes, B. C. Pierce, and L. Xia. 2017. Beginner's luck: a language for property-based generators. In Proc. of the ACM SIGPLAN Symposium on Principles of Programming Languages, POPL.

[19] L. Lampropoulos, Z. Paraskevopoulou, and B. C. Pierce. 2017. Generating Good Generators for Inductive Relations. In Proc. ACM on Programming Languages 2, POPL, Article 45 (2017).

[20] M. Zalewski. 2010. American Fuzzy Lop: a security-oriented fuzzer. http://lcamtuf.coredump.cx/afl/. (2010).

[21] C. McBride and R. Paterson. 2008. Applicative Programming with Effects. Journal of Functional Programming 18, 1 (Jan. 2008).

[22] J. Midtgaard, M. N. Justesen, P. Kasting, F. Nielson, and H. R. Nielson. 2017. Effect-driven QuickChecking of compilers. In Proceedings of the ACM on Programming Languages, Volume 1 ICFP (2017).

[23] A. Mista, A. Russo, and J. Hughes. 2018. Branching Processes for QuickCheck Generators (extended version). https://bitbucket.org/ agustinmista/dragen/downloads/full-paper.pdf. (2018).

[24] N. Mitchell. 2007. Deriving Generic Functions by Example. In Proc. of the 1st York Doctoral Syposium. Tech. Report YCS-2007-421, Department of Computer Science, University of York, UK, 55-62.

[25] M. Pałka, K. Claessen, A. Russo, and J. Hughes. 2011. Testing and Optimising Compiler by Generating Random Lambda Terms. In The IEEE/ACM International Workshop on Automation of Software Test (AST 2011).

[26] S. M. Poulding and R. Feldt. 2017. Automated Random Testing in Multiple Dispatch Languages. IEEE International Conference on Software Testing, Verification and Validation (ICST) (2017).

[27] C. Runciman, M. Naylor, and F. Lindblad. 2008. Smallcheck and Lazy Smallcheck: automatic exhaustive testing for small values. In Proc. of the ACM SIGPLAN Symposium on Haskell.

[28] T. Sheard and Simon L. Peyton Jones. 2002. Template meta-programming for Haskell. SIGPLAN Notices 37, 12 (2002), 60-75.

[29] H. W. Watson and F. Galton. 1875. On the probability of the extinction of families. The Journal of the Anthropological Institute of Great Britain and Ireland (1875). 